\title{
Data Mining Tools and Techniques to Manage the Textile Quality Control Data for Strategic Decision Making
}

\author{
Prof. S.K. Tyagi \\ Professor \& Head, \\ Computer Science \& Engineering, \\ Lord Krishna College of Engineering, \\ Ghaziabad (India) \\ Ph.D. Scholar - Singhania University, India
}

\author{
Dr. B. K. Sharma \\ Sr. Scientific Officer \& Head, \\ Software Development Centre \\ Northern India Textile Research Association \\ Ghaziabad (India)
}

\begin{abstract}
The forces of liberalization, globalization and competition have led to a remarkable evolution in the general theory of quality. Quality control usually refers to an evaluation of inspection, product through testing and recording the nature and analysis of data where necessary, the location of the defect found. In existing the rapid development in Information Technology has ushered in a revolution in manufacturing and interactive marketing across the globe. IT applications like MIS, ERP, Network, Multimedia and Data Mining etc are today's indispensable tools to boost productivity and drive maximum benefits. In other word we can say that the success of an organization depends on faster processing of raw data, which are based on application of Data Mining.
\end{abstract}

The use of Data Mining application to manage quality and laboratory result of the modern textile plant is not a new concept. Most laboratories today have some sort of computerized system to $\log$ and manage data. Every progressive organization has the only major objective to increase its profitability. However, in today's competitive marketplaces, profitability is not only depends on increasing sales but also just as importantly on reducing cost and improving the quality. Introducing Data Mining Tools and Techniques into textile production processes can achieve a substantial increase in productivity and quality of work.

\section{Keywords}

Data Mining, Knowledge Discovery, Quality Control, Information Technology.

\section{INTRODUCTION}

The rapid development in Information Technology has ushered in a revolution in manufacturing and interactive marketing across the globe. IT applications are today's indispensable tools to boost productivity and drive maximum benefits. In other word we can say that the success of an organization depends on faster processing of raw data, which are based on application of information technology. The use of IT application to manage quality and laboratory result of the modern textile plant is not a new concept. Most laboratories today have some sort of computerized system to $\log$ and manage data. Every progressive organization has the only major objective to increase its profitability. However, in today's competitive marketplaces, profitability is not only depends on increasing sales but also just as importantly on reducing cost and improving the quality. Introducing Data Mining Tools and Techniques into textile production processes can achieve a substantial increase in productivity and quality of work. It is here that Data Mining plays a vital role.

\begin{abstract}
Data Mining can be defined as a technique for extracting the "meaning" contained in information to allow the understanding needed by a user to make a "right" decision. Another definition could be providing the right information, in the right form, at the right time, so as to enable the manager to efficiently and effectively perform his/her job. It is Data Mining that allows a computer to digest the constant stream of data being generated by the computerized sensors and monitors of the plant, and then extract from that information that has some meaning content.
\end{abstract}

Data mining tools and techniques can be used for rationalizing the data so as to reduce the overload that tends to occur and make it simple for the personnel to make a right decision in textile industry.

Quality of a product plays an important role in management of an enterprise in order to satisfy customer expectations and to ensure success and survival of a company in a free market economy. The only purpose for any manufacturing activity to exist is to provide a product, which fulfils a known or anticipated need of a consumer. The forces of liberalization, globalization and competition have led to a remarkable evolution in the general theory of quality. Quality control usually refers to an evaluation of inspection, product through testing and recording the nature and analysis of data where necessary, the location of the defect found. This is used to grade the product and if used properly to identify the cause of the defect so an appropriate remedy can be effected. The isolation of problems can have a major effect on the overall quality of products being produced. This can be of on many forms such as mechanical equipment with high defect rate, raw materials and process - dye and finish problems etc. Tracking defects in time can reduce the occurrences and quickly alert management to problem areas.

Customers have become less tolerant to accepting and coping with defects; therefore managing the quality of the products has a direct effect on the bottom line. After all, rejections are expensive, not to mention goodwill to the customer. Defects per unit of measure may be used as selection criteria for any textile item to be used to satisfy a customer order, e.g. total number of imperfections per $\mathrm{km}$ of yarn. Most of the time, a certain value of a particular parameter is compared with the predetermined standard value and used to satisfy the customer, e.g. U\% or RKM values are compared with some international standards like USTER Statistics or fastness of colours are compared with the buyers standard specifications. This requires managing the quality and possibly the defect count or tested value of a parameter in a database to be attached to the finished product record. Documentation of the quality information may be of various forms. The ideal way is to have the inspector directly capture the information on a terminal or other device that can provide real time data. Other 
methods may involve recording the data on a control sheet then entering the data into a computer system. This process is often slow and chances of errors are there. In either case the requirement to capture this information and make the data available quickly is ever increasing.

The textile industry also needs the data from every individual process of every parameter in a compiled form to take prompt and correct decision. In the earlier days this type of information was available from control charts. As for example, if one wants to know the performance of a particular machine in a certain span of time, he has to do a lot of compilation to get certain information. Having the ability to review current quality levels and be able to do something about it before the next day can be a real benefit in terms of time and cost (Dr. Sharma \& Das, 2006).

\section{ROLE OF QUALITY CONTROL IN MODERN TEXTILE INDUSTRY}

The Quality Control department is an essential part of modern textile industry and its important functions are as follows:

1. Evaluation of quality standards of incoming material, products in process and finished goods.

2. Evaluation of optimum quality obtainable under given conditions.

3. Improvement of quality and productivity by process control and experimentation.

The following steps give a summary of the advantages of Q.C. in industry:

- Planned collection of data and analysis

- Improvement in product quality and design

- Reduction in operating cost and losses

- Reduction in scrap/waste

- Saving in excess use of materials

- Reduction in inspection

- Evaluation of scientific tolerance

- Improvement of employee moral

- Maintenance of operating efficiency

- Greater customer satisfaction

The communication chart of Q.C. department, which directly affect different divisions in textile industry, is shown in Fig. 1.

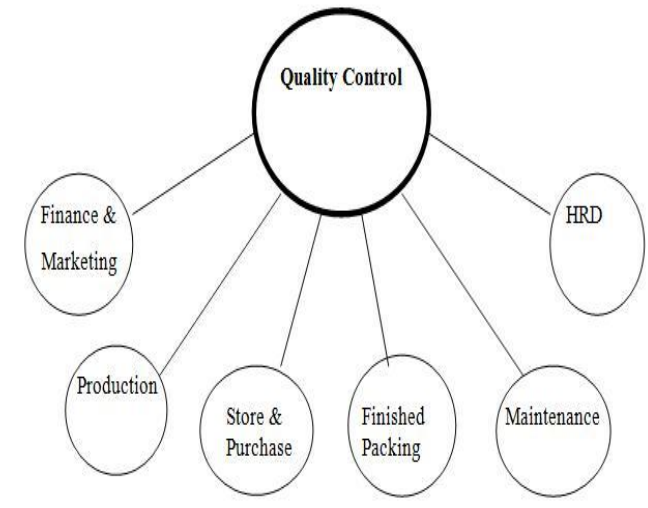

Fig.1: The Communication Chart of QC

\section{IMPORTANCE OF DATA MINING MODEL}

The driving forces behind the need of data mining models can be broken down into two key areas: Understanding and Trust.
Understanding is undoubtedly the most fundamental motivation behind visualizing the model. Although the simplest way to deal with a data mining model is to leave the output in the form of a black box, the user will not necessarily gain an understanding of the underlying behavior in which they are interested. If they take the black box model and score a database, they can get a list of customers to target (send them a catalog, increase their credit limit, etc.). There's not much for the user to do other than sit back and watch the envelopes go out. This can be a very effective approach. Mailing costs can often be reduced by an order of magnitude without significantly reducing the response rate.The more interesting way to use a data mining model is to get the user to actually understand what is going on so that they can take action directly. Visualizing a model should allow a user to discuss and explain the logic behind the model with colleagues, customers, and other users. Getting buy-in on the logic or rationale is part of building the users' trust in the results. For example, if the user is responsible for ordering a print advertising campaign, understanding customer demographics is critical. Decisions about where to put advertising dollars are a direct result of understanding data mining models of customer behavior. There's no automated way to do this. It's all in the marketing manager's head. Unless the output of the data mining system can be understood qualitatively, it won't be of any use. In addition, the model needs to be understood so that the actions that are taken as a result can be justified to others.

\section{ELEMENT AND USES OF DATA MINING IN TEXTILE QUALITY CONTROL}

Generally, data mining is the process of analyzing data from different perspectives and summarizing it into useful information that can be used to increase revenue, cuts costs, or both. Data mining software is one of a number of analytical tools for analyzing data. It allows users to analyze data from many different dimensions or angles, categorize it, and summarize the relationships identified. Technically, data mining is the process of finding correlations or patterns among dozens of fields in large relational databases. Data mining consists of five major elements:

1. Extract, transform, and load transaction data onto the data warehouse system

2. Store and manage the data in a multidimensional database system.

3. Provided data access to business analysts and IT professionals

4. Analyze the data by application software

5. Present the data in a useful format, such as a graph or table.

Different levels of analysis are available:

Artificial neural networks: non-linear predictive models that learn through training and resemble biological neural networks in structure.

Genetic algorithms: Optimization techniques that use processes such as genetic combination, mutation, and selection in a design based on the concepts of natural evolution.

Decision trees: tree- shaped structures that represent sets of decisions. These decisions generate rules for the classification of a dataset. Specific decisions tree methods include 
Classification and regression trees (CART) and chi Square Automatic interaction Detection (CHAID). CART and CHAID are decisions tree techniques used for classification of a dataset. They provide a set of rules that you can apply to a new (unclassified) dataset to predict which records will have a given outcome. CART segments a dataset by creating 2- way splits while CHAID segments using chi square tests to create multi-way splits. CART typically requires less data preparation than CHAID.

Nearest neighbor method: A technique that classifies each record in a data set based on a combination of the classes of the $\mathrm{k}$ record (s) most similar to it in a historical dataset (when $\mathrm{k}=1$ ). Sometimes called the $\mathrm{k}$-nearest neighbors technique.

Rule induction: The extraction of useful if-then rules from data based on statistical significance. Data visualization: The extraction of useful if-then rules from data based on statistical significance.

Data visualization: the visual interpretation of complex relationship in multidimensional data Graphics are used to illustrate data relationships. The point of data visualization is to let the user understand what is going on. Since data mining usually involves extracting "hidden" information from a database, this understanding process can get somewhat complicated. In most standard database operations nearly everything the user sees is something that they knew existed in the database already. A report showing the breakdown of sales by product and region is straightforward for the user to understand because they intuitively know that this kind of information already exists in the database. If the company sells different products in different regions of the county, there is no problem translating a display of this information into a relevant understanding of the business process. The primary benefit of data mining is the ability to turn feeling into facts. Data mining can be used to support or refute feelings of people have about how businesses is going. It can be used to add credibility to these feelings and warrant dedication of more resource and time to the most productive areas of a company's operations. This benefit deals with situations where a company starts the data mining process with an idea of what they are looking for. This is called targeted data mining. Data mining can discover unexpected patterns in behavior, patterns that were not under consideration when the mining exercise commenced. This is called out of the blue data mining (Patricia L. Carbone, 2000).

\section{COMPUTERIZATION IN THE TEXTILE QC LABORATORY}

The use of computers to manage quality and laboratory result of the modern textile plant is not a new concept. Most laboratories today have some sort of computerized system to $\log$ and manage data. These systems are often an integral part of the tests being performed and as such are single application oriented. There are, however, many tests that are required which do not or cannot interface with an automated system and the result are kept in a manual log, maintained in a spreadsheet or entered into a custom application software, specifically designed to be used in the laboratory. The Context-Analysis diagram of the Quality Control Laboratory in Textile Industry is shown in Fig.2.

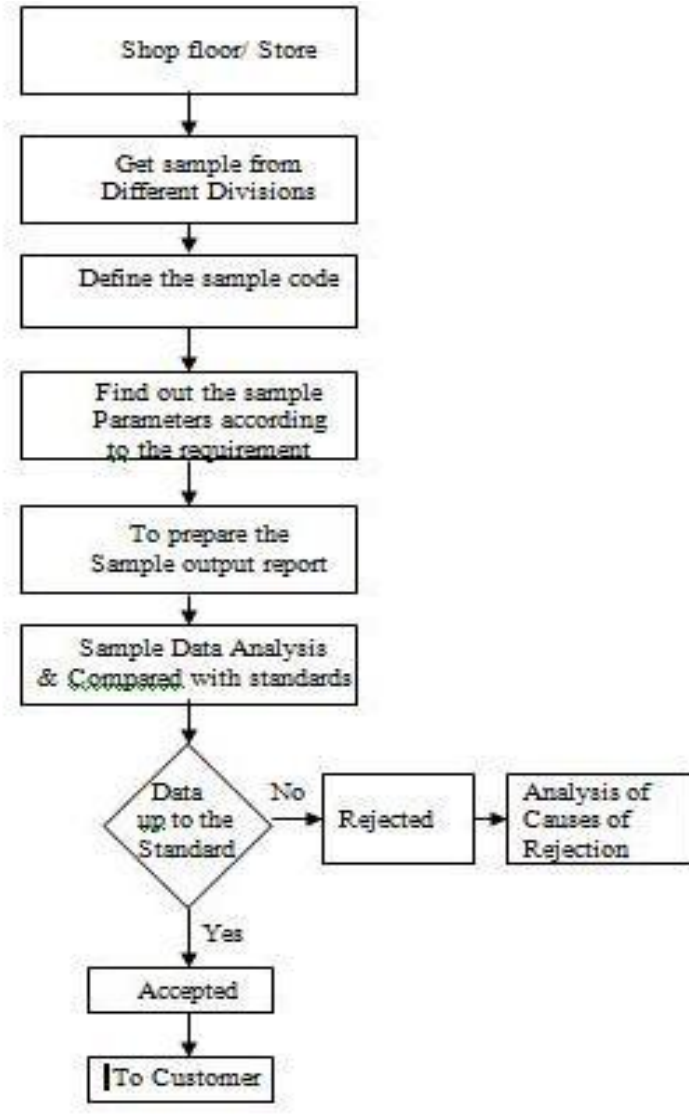

Fig.2: Context-Analysis diagram of the QC Laboratory in Textile Industry

In existing most of the textile industry are maintaining the quality control reports by word processing document/spreadsheets or manually by hand on the paper. Reports generated through word processing document/spreadsheets have enormous disadvantages as compared to tools and techniques of data mining. The some advantages of data mining tools \& techniques in textile quality control are: The data cannot be accessed at once.

- Backup data cannot be accessed and summarized by a key press.

- Data analysis and graphical presentation cannot view by a key press.

- $\quad$ Large number of data, e.g. one billion, cannot be handled by the existing systems.

- Past data cannot be stored and accessed by key press.

- Quality control automation and Management decision making for Q.C. parameters.

- Helpful in taking quick decision regarding product development and data analysis.

- $\quad$ Fibre/Yarn test parameters report.

- Process wise breakage data analysis.

- $\quad$ Process -wise test report.

- Machine wise Process parameters.

- Process wise Graphical analysis

- Customer Complain Analysis report

- Comparison reports over standard norms.

- Systematic and smooth flow information in the functional area.

- Quick compilation and analysis of large volume of data from various departments helps timely decision 
making, thus results reduction in unwanted defective products.

- Helps the management to decide upon the marketing and product mix strategies.

- Better control over the system.

Main advantage of this technique is comparing the various QC parameters to International Norms, TRA's Norms and DM Norms. DM Norms is based on daily QC parameters and calculated by data Mining Techniques. The graphical representation of machine wise graphical analysis of blow room (Lap CV\%) as shown in Fig.3.

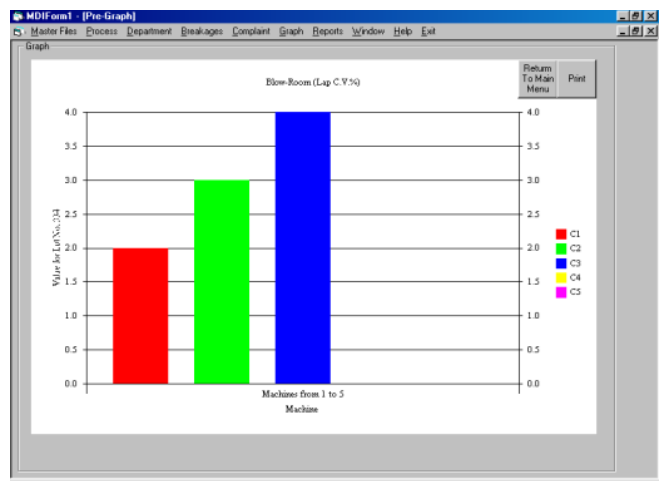

Fig.3: M/c Wise graphical Analysis of Blow Room (Lap CV \%)

\section{CONCLUSION}

Quality and the laboratory go hand in hand. Quality can be improved without any extra cost, by constant monitoring the laboratory test data, analyzing them in a scientific manner, take right decision on the basis of analyzed data in right time. Today's Data Mining tools and techniques allows for flexibility, rapid availability of information and timely response. The proper managing the laboratory data not only helps in improving the quality level, but also reduce the losses due to defective production. The proper use and application of the laboratory data is very important factor in the whole process of manufacturing. The better the way the laboratory test data are handled, the better will be the performance of an industry by the way of less defects, improved quality, better performance and overall customer satisfaction.

\section{REFERENCES}

[1] Dr. B.K. Sharma, D.K. Sharma, Application of Information Technology in Textile Wet Processing for Strategic Decision Making, International Journal of Management and System, Australia

[2] Prof. S.M. Ishtiaque, Dr. B.K. Sharma, D.K. Sharma, Management Information System in Textile Wet Processing for batter decision-making International Conference on IT in Textile Sector organized by Ministry of Textile, Govt. of India

[3] Dr. A.K. Srivastava, Dr. B.K. Sharma and R.S. Yadav, Marketing Information System in Textile Sector, The Indian Textile Journal, May, 2001
[4] Dr. B.K. Sharma \& Dr. A. Das, Application of information Technology to manage the textile quality control text data, The Textile Industry and Trade Journal, Vol. No. 44, No. 5-6, May-June 2006

[5] Patricia L. Carbone Expanding the Meaning of and Applications for Data Mining 2000 IEEE

[6 ] Dr. B.K. Sharma, IT: A Benison for Industry presented at 5th International Petroleum Conference (PETROTACH 2003) organized by Ministry of Petroleum and Natural Gas, Govt. of India

[7] Soler, S. and D. Yankelevich (2001). "Quality Mining: A data Mining Based Method for Data Quality Evaluation", Processing of the Sixth international Conference on Data Quality, MIT.

[8] Dongsong Zhang and Lina Zhou Discovering Goden Nuggets: Data Mining in Financial Application 2004 IEEE.

[9] Zhen LIU, Minyi GUO A Proposal of Integrating Data Mining and On-Line Analytical Processing in Data Warehouse 2001 IEEE.

[10] Ruoming Jin, Ge Yang, and Gagan Agrawal, Shared Memory Parallelization of Data Mining lgorithms: Techniques, Programming Interface, and Performance IEEE 2005

[11] Dr. A.K. Srivastava, Dr. B.K. Sharma and R.K. Gaur, Application of IT for integrating organization information to meet out competitive business environment for textile spinning mills presented at $45^{\text {th }}$ Joint Technology Conference, BTRA, Mumbai, India

[12] D.K. Bhatachariya, Dr. B.K. Sharma, Sanjeev Saxena 'Evaluation of Energy and Monitoring software for fuel conservation in Textile' at 3rd International Symposium on fuels and lubricants (ISFL-2002) IOC, Govt. of India

[13] Dr. B.K. Sharma, Prof. S.K. \& Abhay Bansal Data Mining Tools and Techniques in Textile Industry for Effective Decision Making and Corrective Action, Asian Textile Journal, Vol No. 15, No. 8, August 2006

[14] C. Brunk, J. Kelly, and R. Kohavi, "MineSet: An Integrated System for Data Access, Visual Data Mining, and Analytical Data Mining," Proceedings of the Third Conference on Knowledge Discovery and Data Mining (KDD-97), Newport Beach, CA, August 1997.

[15] G.K. Gupta, A book on introduction to Data Mining with Case Studies 